\title{
Editorial
}

\author{
Aldo Clerico*, Martina Zaninotto and Mario Plebani
}

\section{High-sensitivity assay for cardiac troponins with POCT methods. The future is soon}

\section{https://doi.org/10.1515/cclm-2021-0620}

Keywords: acute coronary syndrome; cardiac troponins; guidelines; high-sensitivity immunoassay; myocardial infarction; POCT methods; quality specification; reference population.

A well-known mantra highlights that "good, fast and cheap" in laboratory testing is a mission impossible. However, the recent history of cardiac troponin assays may deny this aphorism. In the last 10 years, the introduction of high-sensitivity cardiac troponin (hs-cTn) assays made it possible to progressively reduce the time to diagnosis of acute myocardial infarction (AMI) from 6 to $12 \mathrm{~h}$ to from 1 to $3 \mathrm{~h}[1,2]$. In particular, the $2020 \mathrm{ESC}$ guidelines recommend the rapid 0/1-h algorithm (blood draw at admission at baseline and $1 \mathrm{~h}$ later) as the first clinical option because it provides the best balance between safety and efficacy by allowing the reduction of the length of stays in Emergence Departments (ED) of patients with suspect of Acute Coronary Syndromes (ACS) [3].

Until now, the successful clinical implementation of these rapid algorithms was restricted to clinical hospital laboratories, as current hs-cTn methods require the use of large automated platforms [3, 4]. Point-of-care-testing (POCT) for cTn assays with high-sensitivity performance may represent a formidable progress because they should assure a more rapid diagnostic turnaround-time, thus facilitating the valuable management of patients admitted to Emergency Departments with chest pain [3]. Furthermore, hs-cTn POCT assays may open the possibility of a "decentralized" diagnosis of myocardial injury and infarction, even in primary care and other remote clinical settings [5]. However, most commercially available POCT

*Corresponding author: Aldo Clerico, MD, Professor, Department of Laboratory Medicine, Cardiovascular Endocrinology and Cell Biology, Scuola Superiore Sant'Anna e Fondazione CNR - Regione Toscana G. Monasterio, Via Trieste 41, 56126 Pisa, Italy, E-mail: clerico@ftgm.it Martina Zaninotto, Department of Laboratory Medicine, University Hospital of Padova, Padova, Italy

Mario Plebani, Department of Laboratory Medicine, University Hospital of Padova, Padova, Italy; and Department of MedicineDIMED, University of Padova, Padova, Italy methods for cTn, did not meet the criteria recommended to be considered an high-sensitivity assay [3, 4, 6]. Therefore, the obvious advantage of POCT methods, namely the shorter turnaround-time, was counterbalanced by lower sensitivity, lower diagnostic accuracy and lower negative predictive value [3].

However, very recent studies suggest that POCT methods for cTn could provide comparable analytical performance characteristics to those of central laboratory hscTn assays. In 2019, Sorensen et al. [7] reported the clinical results obtained with the PATHFAST POC hs-cTnI assay using the PATHFAST immunoanalyzer system, which is a POCT method with analytical performance compliant with an high-sensitivity assay. Diagnostic algorithms using rapid $(0 / 1 \mathrm{~h})$ and standard $0 / 3 \mathrm{~h}$ approaches were evaluated in a derivation data set with 669 patients and validated in an additional 610 patients and compared with those obtained with the Architect hs-cTnI method [7]. Moreover, in 2020 Boeddinghaus et al. [8] directly compared the diagnostic accuracy of POC-hs-cTnI-TriageTrue vs. the best-validated central laboratory assays using specific $0 / 1$-h algorithms in 1,261 patients (178 with AMI, 14\%). The POC-hs-cTnITriageTrue assay provided high diagnostic accuracy in patients with suspected AMI with a clinical performance comparable to that of the best-validated central laboratory assays [8].

In this issue of the Journal, Apple et al. [9] report the sex-specific 99th percentile URL values for males and females in heparinized plasma from the AACC universal sample bank (USB) using the Siemens point of care (POC) Atellica VTLi hs-cTnI immunoassay. The reference population (age range: $18-91$ years, median: 39 years) included 693 subjects, 363 males and 330 females [9]. Of note, Authors excluded the possible outliers using some surrogate biomarkers, also including NT-proBNP, as recommended by international guidelines and some expert documents [10-13]. The calculated 99th percentile URL values were: overall, $23 \mathrm{ng} / \mathrm{L}$ [90\% confidence interval (CI)] 20-32 ng/L; males, $27 \mathrm{ng} / \mathrm{L}$ (90\% CI 21-37 ng/L); females, $18 \mathrm{ng} / \mathrm{L}$ (90\% CI 9-78 ng/L) [9]. The percentages of subjects having measurable concentrations higher than LoD for the post exclusion subjects were: overall $83.7 \%$, 
male $87.3 \%$, female $79.7 \%$ with no statistical differences compared to the non-exclusion subjects [9]. The results of this study shows that the novel POC Atellica ${ }^{\circledR}$ VTLi hscTnI assay meets the designation of a 'high-sensitivity' assay according to the criteria recommended by current international guidelines [10].

As for the development of reliable POCT tests for the Pandemic COVID-19 disease [14], the progression toward reliable POCT methods for hs-cTn assay to reach the marriage between quickness and consistency was certainly not an easy task. As principles of Laboratory Medicine state, speed and accuracy should go hand in hand and the presumed advantages of rapid testing need to be balanced against the possible lower sensitivity (sometimes even specificity) [14].

As noted by the NICE guidelines [4], the major limitation of recent clinical studies on hs-cTnI POCT methods [7-9] is that troponin levels were tested in stored plasma samples rather than in whole blood samples used in clinical practice, the so-called "real life". Very recently, Gopi et al. [15] investigated the agreement between plasma and whole blood hs-cTnI by using the PATHFAST hs-cTnI assay. Furthermore, these Authors compared the results of POCT method with those measured in the central laboratory with the hs-cTnT assay in 224 fresh samples collected from 191 patients presenting with suspected ACS. The results of this study indicate that whole blood can be used interchangeably with plasma for a more convenient and less time- and labor-consuming testing of hs-cTnI on the PATHFAST analyzer [15].

According to the NICE guidelines, therefore, we may conclude that further evidence on diagnostic performances of POCT hs-cTnI methods is needed before these tests can be recommended for the use in clinical practice [4]. However, taking as a whole, the most recently available data $[7-9,15]$ strongly suggest that for POCT high-sensitivity methods to measure cTn, "the future is soon", even if some further steps should be taken before crossing the finish line.

Research funding: None declared.

Author contributions: All authors have accepted responsibility for the entire content of this manuscript and approved its submission.

Competing interests: Authors state no conflict of interest.

\section{References}

1. Clerico A, Zaninotto M, Padoan A, Masotti S, Musetti V, Prontera C, et al. Evaluation of analytical performance of immunoassay methods for cardiac troponin I and T: from theory to practice. Adv Clin Chem 2019;93:239-62.
2. Collinson P. High sensitivity troponin, analytical advantages, clinical benefits and clinical challenges-an update. Clin Biochem 2021;91:1-8.

3. Collet JP, Thiele H, Barbato E, Barthélémy O, Bauersachs J, Bhatt DL, et al. 2020 ESC Guidelines for the management of acute coronary syndromes in patients presenting without persistent ST-segment elevation. Eur Heart J 2021;42:1289-367.

4. Body R, Collinson P, Mills N, Reid A, Timmis A. Diagnostics guidance [DG40] Diagnostics Assessment Committee National Institute for Health and Care Excellence. High-sensitivity troponin tests for the early rule out of NSTEMI. NICE; 2020:1-42. https:// www.nice.org.uk/guidance/DG40.

5. Schols AMR, Stakenborg JPG, Dinant GJ, Willemsen RTA, Cals JWL. Point-of-care testing in primary care patients with acute cardiopulmonary symptoms: a systematic review. Fam Pract 2018;35:4-12.

6. Collinson PO, Saenger AK, Apple FS, IFCC C-CB. High sensitivity, contemporary and point-of-care cardiac troponin assays: educational aids developed by the IFCC Committee on Clinical Application of Cardiac Bio-Markers. Clin Chem Lab Med 2019;57: 623-32.

7. Sörensen NA, Neumann JT, Ojeda F, Giannitsis E, Spanuth E, Blankenberg S, et al. Diagnostic evaluation of a high-sensitivity troponin I point-of-care assay. Clin Chem 2019;65:1592-601.

8. Boeddinghaus J, Nestelberger T, Koechlin L, Wussler D, LopezAyala P, Walter JE, et al. Early diagnosis of myocardial infarction with Point-of-Care high-sensitivity cardiac troponin I. J Am Coll Cardiol 2020;75:1111-24.

9. Apple FS, Schulz K, Schmidt CW, Van Domburg TSY, Fonville JM, de Theije FK. Determination of sex-specific 99th percentile upper reference limits for a point of care high sensitivity cardiac troponin I assay. Clin Chem Lab Med 2021;59:1574-8.

10. Wu AHB, Christenson RH, Greene DN, Jaffe AS, Kavsak PA, Ordonez-Lianos J, et al. Clinical laboratory practice recommendations for the use of cardiac troponin in acute coronary syndrome: expert opinion from the Academy of the American Association for clinical Chemistry and the Task Force on clinical Applications of cardiac Bio-Markers of the international Federation of Clinical Chemistry and laboratory Medicine. Clin Chem 2018;64:645-55.

11. Sandoval Y, Apple FS. The global need to define normality: the 99th percentile value of cardiac troponin. Clin Chem 2014;60: 455-62.

12. Clerico A, Zaninotto M, Ripoli M, Masotti S, Prontera C, Passino C, et al. The 99th percentile of reference population for $\mathrm{CTnI}$ and $\mathrm{CTnT}$ assay: methodology, pathophysiology, and clinical implications. Clin Chem Lab Med 2017;55:1634-51.

13. Clerico A, Ripoli A, Zaninotto M, Masotti S, Musetti V, Ciaccio M, et al. Head-to-head comparison of plasma cTnl concentration values measured with three high-sensitivity methods in a large Italian population of healthy volunteers and patients admitted to emergency department with acute coronary syndrome: a multicenter study. Clin Chim Acta 2019;496:25-34.

14. Plebani M. Laboratory medicine in the COVID-19 era: six lessons for the future. Clin Chem Lab Med 2021;59:1035-45.

15. Gopi V, Milles B, Spanuth E, Müller-Hennessen M, Biener M, Stoyanov K, et al. Comparison of the analytical performance of the PATHFAST high sensitivity cardiac troponin I using fresh whole blood versus fresh plasma samples. Clin Chem Lab Med 2021;59:1579-84. 